The Astrophysical Journal, 572:L103-L107, 2002 June 10

(C) 2002. The American Astronomical Society. All rights reserved. Printed in U.S.A.

\title{
INTERACTING CORONAL MASS EJECTIONS AND SOLAR ENERGETIC PARTICLES
}

\author{
N. Gopalswamy, ${ }^{1}$ S. Yashiro, ${ }^{1,2}$ G. MichaŁeK, ${ }^{1,2}$ M. L. Kaiser,${ }^{1}$ R. A. Howard, ${ }^{3}$ D. V. Reames, ${ }^{1}$ \\ R. LESKe, ${ }^{4}$ AND T. VON Rosenvinge ${ }^{1}$ \\ Received 2002 February 3; accepted 2002 May 7; published 2002 May 16
}

\begin{abstract}
We studied the association between solar energetic particle (SEP) events and coronal mass ejections (CMEs) and found that CME interaction is an important aspect of SEP production. Each SEP event was associated with a primary CME that is faster and wider than average CMEs and originated from west of E45. For most of the SEP events, the primary CME overtakes one or more slower CMEs within a heliocentric distance of $\sim 20 R_{\odot}$. In an inverse study, we found that for all the fast (speed greater than $900 \mathrm{~km} \mathrm{~s}^{-1}$ ) and wide (width greater than $60^{\circ}$ ) western hemispheric frontside CMEs during the study period, the SEP-associated CMEs were $\sim 4$ times more likely to be preceded by CME interaction than the SEP-poor CMEs; i.e., CME interaction is a good discriminator between SEP-poor and SEP-associated CMEs. We infer that the efficiency of the CME-driven shocks is enhanced as they propagate through the preceding CMEs and that they accelerate SEPs from the material of the preceding CMEs rather than from the quiet solar wind. We also found a high degree of association between major SEP events and interplanetary type II radio bursts, suggesting that proton accelerators are also good electron accelerators.
\end{abstract}

Subject headings: solar-terrestrial relations — Sun: corona — Sun: coronal mass ejections (CMEs) Sun: particle emission — Sun: radio radiation

\section{INTRODUCTION}

Large solar energetic particle (SEP) events are known to be closely related to coronal mass ejections (CMEs) (see, e.g., Reames 1999). Fast CMEs drive MHD shocks, which in turn accelerate the SEPs (protons and minor ions). SEP acceleration may also occur during flares, but these events are typically short-lived (hours). For space weather purposes, the large gradual SEPs from CME-driven shocks are more important. More than 4500 CMEs were observed from 1996 January to the end of 2001, ${ }^{5}$ yet only $~ 100$ SEP events with $I_{p}$ (intensity of more than $10 \mathrm{MeV}$ protons) exceeding 1 particle flux unit (pfu) (=1 particle $\mathrm{cm}^{-2} \mathrm{~s}^{-1} \mathrm{sr}^{-1}$ ) were observed during the same period. Thus only a small fraction (1\%-2\%) of CMEs is associated with SEPs, and it is important to know what makes a CME an SEP accelerator. It is known that the CME speed and the SEP intensity are well correlated (Kahler 2001). Faster and wider CMEs produce decameter-hectometric (DH) type II radio bursts, so they must be good electron accelerators (Gopalswamy et al. 2001b). Recently, Gopalswamy et al. (2001c, 2002) found that interaction between CMEs can influence the production of nonthermal electrons, as inferred from intense radio emission in the interplanetary (IP) medium. In this Letter, we investigate whether CME interactions are also important for SEP production.

\section{DATA SELECTION AND ANALYSIS}

From GOES proton data, we identified 43 major $\left(I_{p} \geq 10 \mathrm{pfu}\right)$ and 39 minor (1 pfu $\leq I_{p}<10 \mathrm{pfu}$ ) SEP events from 20011996 January to November 4 by requiring that simultaneous data exist for SEPs and CMEs. The CMEs were observed by the Solar

\footnotetext{
${ }^{1}$ LEP, NASA Goddard Space Flight Center, Greenbelt, MD 20771.

${ }^{2}$ Center for Solar Physics and Space Weather, Catholic University of America, Washington, DC 20064.

${ }^{3}$ Naval Research Laboratory, Washington, DC 20375.

${ }^{4}$ California Institute of Technology, Mail Code 220-47, Pasadena, CA 91125.

${ }^{5}$ See the CME catalog at http://cdaw.gsfc.nasa.gov.
}

and Heliospheric Observatory ( $\mathrm{SOHO}$ ) mission's Large Angle and Spectrometric Coronagraphs (LASCO; Brueckner et al. 1995) known as C2 and C3. Each SEP event corresponds to a unique white-light $\mathrm{CME}$, which we call the primary $\mathrm{CME}$, as listed in Table 1. In addition to the SEP date (col. [1]) and onset time (col. [2]), the CME onset (col. [3]), speed ( $V$, in col. [4]), width ( $W$, in col. [5]), and the heliographic coordinates of the solar sources (col. [6]) are also listed. The solar sources were obtained from the on-line Solar Geophysical Data (SGD) as the location of the associated $\mathrm{H} \alpha$ flare. When $\mathrm{H} \alpha$ information is not available, we used movies from the Extreme-ultraviolet Imaging Telescope (EIT) on board $\mathrm{SOHO}$ and the Yohkoh mission's soft $\mathrm{X}$-ray telescope to identify the location of the eruption. For one major event (2001 August 9), we identified the location of the eruption $\left(\mathrm{S}^{\circ} 7^{\circ}, \mathrm{E} 19^{\circ}\right)$ from EIT images, but the CME could not be measured. For some events, the solar sources were behind the west limb (noted as backside or (b) in col. [6]). Whether or not an SEP event was associated with a type II burst in the metric ("y" for yes and " $\mathrm{n}$ " for no) and DH ("Y" for yes and "N" for no) wavelengths is noted in column (7). Information on the metric type II bursts were obtained from SGD. The DH type II bursts were observed by the Radio and Plasma Wave Experiment (WAVES; Bougeret et al. 1995) on board the Wind spacecraft.

Height-time plots of CMEs were extended to 30 and $50 R_{\odot}$ to see if the trajectories of the preceding CMEs intersect that of the primary CME. We also required that the preceding CMEs with intersecting trajectories have a position angle (P.A.) overlap $(\Delta w)$ with the primary CMEs. We designated the interaction to be full (F) when $\Delta w>30^{\circ}$ and partial (P) when $\Delta w<30^{\circ}$. We also played movies of LASCO images to visually examine and confirm the physical overlap. Events with height-time overlap but no P.A. overlap were eliminated. The extent of CME interaction is listed in column (8) (Int) of Table 1: F and P are suffixed by the number of CMEs interacting with the primary CME. When the intersection of trajectories occur between 30 and $50 R_{\odot}$, we added a question mark to indicate that the interaction may be less severe. Some events had both F and P interactions (marked by footnote $\mathrm{f}$ in cols. [8] and [16]). In a 
TABLE 1

Properties of Primary CME Associated with Major and Minor SEP Events

\begin{tabular}{|c|c|c|c|c|c|c|c|c|c|c|c|c|c|c|c|}
\hline \multicolumn{8}{|c|}{ MAJOR } & \multicolumn{8}{|c|}{ MINOR } \\
\hline \multirow[b]{2}{*}{$\begin{array}{l}\text { Date } \\
(1)\end{array}$} & \multirow{2}{*}{$\begin{array}{c}\text { SEP } \\
\begin{array}{c}\text { Time } \\
(2)\end{array}\end{array}$} & \multicolumn{6}{|c|}{$\mathrm{CME}$} & \multirow[b]{2}{*}{$\begin{array}{l}\text { Date } \\
(9)\end{array}$} & \multirow{2}{*}{$\begin{array}{l}\text { SEP } \\
\text { Time } \\
(10)\end{array}$} & \multicolumn{6}{|c|}{ CME } \\
\hline & & $\begin{array}{l}\text { Time } \\
(3)\end{array}$ & $\begin{array}{l}V^{\mathrm{a}} \\
(4)\end{array}$ & $\begin{array}{l}W^{\mathrm{b}} \\
(5)\end{array}$ & $\begin{array}{l}\text { Location } \\
\text { (deg) } \\
\text { (6) }\end{array}$ & $\begin{array}{l}\mathrm{II}^{\mathrm{c}} \\
(7)\end{array}$ & $\begin{array}{l}\mathrm{Int}^{\mathrm{d}} \\
(8)\end{array}$ & & & $\begin{array}{l}\text { Time } \\
\text { (11) }\end{array}$ & $\begin{array}{c}V^{\mathrm{a}} \\
(12)\end{array}$ & $\begin{array}{c}W^{\mathrm{b}} \\
(13)\end{array}$ & $\begin{array}{l}\text { Location } \\
\text { (deg) } \\
(14)\end{array}$ & $\begin{array}{c}\mathrm{II}^{\mathrm{c}} \\
(15)\end{array}$ & $\begin{array}{l}\text { Int }^{d} \\
(16)\end{array}$ \\
\hline 1997 Nov 4 & 07:00 & $06: 10$ & 785 & $\mathrm{H}$ & S14, W33 & $\mathrm{yY}$ & F1 & 1997 Apr 7 & $12: 00$ & $14: 27$ & 878 & $\mathrm{H}$ & S30, E19 & $\mathrm{yY}$ & $\mathrm{F} 1$ \\
\hline 1997 Nov 6 & $13: 00$ & $12: 10$ & 1556 & $\mathrm{H}$ & S18, W63 & yY & F1 & 1997 May 12 & 04:00 & $06: 30$ & 464 & $\mathrm{H}$ & N21, W08 & $\mathrm{yY}$ & $\mathrm{P} 1$ \\
\hline 1998 Apr 20 & $11: 00$ & 10:07 & 1863 & 165 & S43, W90 & yY & F1 & 1997 Nov 13 & 23:00 & $22: 25$ & 546 & 288 & NW90(b) & $\mathrm{nN}$ & $\mathrm{F} 2$ \\
\hline 1998 May 2. & $14: 00$ & $14: 06$ & 938 & $\mathrm{H}$ & S15, W15 & yY & $\mathrm{NH}$ & 1997 Nov 14 & 14:00 & $13: 36$ & 702 & 217 & N22, W110 & $\mathrm{nN}$ & NS \\
\hline 1998 May $6 \ldots \ldots$ & 08:00 & 08:29 & 1099 & 190 & S11, W65 & yY & $F 1^{f}$ & 1998 Apr 30 & 00:00 & $16: 58^{\mathrm{e}}$ & 1374 & $\mathrm{H}$ & S18, E20 & yY & $\mathrm{F} 1^{\mathrm{f}}$ \\
\hline 1998 May $9 \ldots \ldots$. & 05:00 & $03: 35$ & 2331 & 178 & S11, W90 & yY & $\mathrm{F} 1$ & 1998 Jun 4 & 09:00 & 02:04 & 1802 & $\mathrm{H}$ & NW90(b) & $\mathrm{nN}$ & P3 \\
\hline 1999 Apr 24 & $15: 00$ & $13: 31$ & 1495 & $\mathrm{H}$ & NW90(b) & nY & $\mathrm{F} 2 ?$ & 1998 Jun 16 & 21:00 & $18: 27$ & 1484 & 281 & $\mathrm{~S} 17, \mathrm{~W} 90$ & yY & F1? \\
\hline 1999 May 3 ....... & 13:00 & $06: 06$ & 1584 & $\mathrm{H}$ & N15, E32 & yY & F3 & 1999 Мау 9 & 19:00 & $18: 27$ & 615 & 172 & N26, W90 & $\mathrm{nN}$ & $\mathrm{F} 1$ \\
\hline 1999 Jun 1 & $20: 00$ & 19:37 & 1772 & $\mathrm{H}$ & NW90(b) & nY & F1 & 1999 May 27 & $12: 00$ & $11: 06$ & 1691 & $\mathrm{H}$ & NW90(b) & yY & NS \\
\hline 1999 Jun 4 & 08:00 & $07: 26$ & 2230 & 150 & N17, W69 & yY & F1? & 1999 Jun 11 & 01:00 & $01: 26$ & 719 & 101 & SW90(b) & yN & $\mathrm{F} 1$ ? \\
\hline 2000 Feb 18 & $10: 00$ & 09:54 & 890 & 118 & S16, W78 & yY & F1? & 2000 Jan 18 & 19:00 & $17: 54$ & 739 & $\mathrm{H}$ & S19, E11 & yY & $\mathrm{F} 2$ \\
\hline 2000 Apr 4. & $17: 00$ & $16: 32$ & 1188 & $\mathrm{H}$ & N16, W66 & yY & $\mathrm{F} 2$ & 2000 Feb 12 & 06:00 & 04:31 & 1107 & $\mathrm{H}$ & N26, W23 & yY & $\mathrm{F} 1^{\mathrm{f}}$ \\
\hline 2000 Jun 6 & 19:00 & $15: 54$ & 1119 & $\mathrm{H}$ & N20, E18 & yY & F3 & 2000 Feb 17 & $22: 00$ & $20: 06$ & 600 & $\mathrm{H}$ & S29, E07 & yY & $\mathrm{F} 1^{\mathrm{f}}$ \\
\hline 2000 Jun 10 & $18: 00$ & $17: 08$ & 1108 & $\mathrm{H}$ & $\mathrm{N} 22, \mathrm{~W} 38$ & yY & $\mathrm{F} 4^{\mathrm{f}}$ & 2000 Mar 22 & 19:00 & 19:31 & 478 & 93 & N14, W57 & yN & $\mathrm{F} 1$ ? \\
\hline 2000 Jul 14 & $11: 00$ & $10: 54$ & 1674 & $\mathrm{H}$ & N22, W07 & yY & $\mathrm{F} 2$ & 2000 Apr 23 & 15:00 & $12: 54$ & 1187 & $\mathrm{H}$ & N12, W90 & $\mathrm{nN}$ & $\mathrm{N}$ \\
\hline 2000 Jul 22 & $12: 00$ & $11: 54$ & 1230 & 105 & N14, W56 & yY & $F 1^{f}$ & 2000 May 15 & 18:00 & $16: 26$ & 1212 & 165 & S24, W67 & $\mathrm{nY}$ & F1 \\
\hline $2000 \mathrm{Jul} 28$ & 01:00 & $19: 54^{\mathrm{e}}$ & 905 & $\mathrm{H}$ & Backside & $\mathrm{nN}$ & $\mathrm{N}$ & 2000 May 17. & 19:00 & $19: 26$ & 777 & 109 & S22, W37 & $\mathrm{nN}$ & $\mathrm{P} 1$ \\
\hline 2000 Aug $11 \ldots \ldots$ & $12: 00$ & 07:31 & 1071 & 70 & $\mathrm{~N} 27, \mathrm{~W} 90$ & $\mathrm{nN}$ & P1 & 2000 Jun 17 & 03:00 & $03: 28$ & 857 & 133 & $\mathrm{~N} 22, \mathrm{~W} 72$ & $\mathrm{nY}$ & $\mathrm{F} 1$ ? \\
\hline 2000 Sep $12 \ldots \ldots$ & 13:00 & $11: 54$ & 1550 & $\mathrm{H}$ & S17, W09 & yY & $\mathrm{F} 1^{\mathrm{f}}$ & 2000 Jun 18 & 03:00 & $02: 10$ & 629 & 132 & $\mathrm{~N} 23, \mathrm{~W} 85$ & $\mathrm{yN}$ & NL \\
\hline 2000 Oct $16 \ldots \ldots$ & 08:00 & $07: 27$ & 1336 & $\mathrm{H}$ & N05, W90 & yY & $F 3^{\mathrm{f}}$ & 2000 Jun 23 & 16:00 & $14: 54$ & 847 & 103 & N26, W72 & yY & $\mathrm{F} 1$ \\
\hline 2000 Oct $25 \ldots \ldots$ & $12: 00$ & $08: 26$ & 770 & $\mathrm{H}$ & N10, W66 & $\mathrm{nY}$ & F1? & 2000 Jun 25 & 10:00 & $07: 54$ & 1617 & 165 & N16, W55 & yN & P1 \\
\hline 2000 Nov 8 & 23:00 & 23:06 & 1345 & $\mathrm{H}$ & N10, W77 & $\mathrm{nY}$ & $\mathrm{F} 2$ & $2000 \mathrm{Jul} 11$ & 14:00 & $13: 27$ & 1078 & $\mathrm{H}$ & N18, E27 & $\mathrm{nY}$ & $\mathrm{F} 2^{\mathrm{f}}$ \\
\hline 2000 Nov $24 \ldots \ldots$ & $14: 00$ & $15: 30$ & 1245 & $\mathrm{H}$ & $\mathrm{N} 22, \mathrm{~W} 07$ & yY & $F 2^{f}$ & $2000 \mathrm{Jul} 12$ & 19:00 & $20: 30$ & 820 & 101 & N16, W64 & yY & $\mathrm{F} 1$ \\
\hline 2000 Nov $26 \ldots \ldots$ & 06:00 & $06: 30$ & 984 & 227 & NW90(b) & $\mathrm{nY}$ & $\mathrm{F} 1$ & 2000 Aug 13 & 06:00 & 06:06 & 883 & 154 & NW90(b) & $\mathrm{nN}$ & $\mathrm{F} 1$ \\
\hline 2000 Nov $26 \ldots \ldots$ & $17: 00$ & $17: 06$ & 980 & $\mathrm{H}$ & N18, W38 & yY & NL & 2000 Sep 19 & $14: 00$ & 08:50 & 766 & 76 & $\mathrm{~N} 14, \mathrm{~W} 46$ & yY & $F 1^{\mathrm{f}}$ \\
\hline 2001 Jan $28 \ldots \ldots$. & $17: 00$ & $15: 54$ & 916 & 250 & S04, W59 & $\mathrm{nY}$ & $\mathrm{N}$ & 2000 Nov 4 & $03: 30$ & $01: 50$ & 763 & 100 & S23, W75 & $\mathrm{nN}$ & $\mathrm{F} 1$ \\
\hline 2001 Mar 29 & $11: 00$ & $10: 26$ & 942 & $\mathrm{H}$ & $\mathrm{N} 20, \mathrm{~W} 19$ & yY & F1 & 2000 Nov 24 & 06:00 & $05: 30$ & 994 & $\mathrm{H}$ & N20, W05 & yY & $\mathrm{F} 2$ \\
\hline 2001 Apr $2 \ldots \ldots \ldots$ & 23:00 & $22: 06$ & 2505 & 244 & N19, W72 & yY & F1? & 2000 Dec $28 \ldots \ldots$ & $16: 00$ & $12: 06$ & 930 & $\mathrm{H}$ & Backside & $\mathrm{nN}$ & $\mathrm{F} 2$ \\
\hline 2001 Apr 10 & 08:00 & $05: 30$ & 2411 & $\mathrm{H}$ & S23, W09 & yY & $\mathrm{F} 2$ & $2001 \mathrm{Jan} 5 \ldots$ & 19:00 & $17: 06$ & 828 & $\mathrm{H}$ & SW90(b) & $\mathrm{nN}$ & $\mathrm{F} 1^{\mathrm{f}}$ \\
\hline 2001 Apr $12 \ldots \ldots$ & $12: 00$ & $10: 31$ & 1184 & $\mathrm{H}$ & S19, W43 & yY & $\mathrm{N}$ & 2001 Jan 21 & 20:00 & $21: 30^{\mathrm{e}}$ & 1507 & $\mathrm{H}$ & S07, E46 & $\mathrm{yY}$ & $\mathrm{F} 1$ \\
\hline 2001 Apr 15 & $14: 00$ & $14: 06$ & 1199 & 167 & S20, W85 & yY & F3 & 2001 Feb 11 & 03:00 & 01:31 & 1183 & $\mathrm{H}$ & N24, W57 & yY & F3 \\
\hline 2001 Apr $18 \ldots \ldots$ & 03:00 & $02: 30$ & 2465 & $\mathrm{H}$ & $\mathrm{W} 120$ & yY & NS & 2001 Feb 26 & 08:00 & $05: 30$ & 851 & 152 & NW90(b) & nY & NS \\
\hline 2001 Apr $26 \ldots \ldots$ & $14: 00$ & $12: 30$ & 1006 & $\mathrm{H}$ & N17, W31 & yY & F1? & 2001 Mar $25 \ldots \ldots$ & 15:00 & 17:06 & 677 & $\mathrm{H}$ & N16, E25 & $\mathrm{nN}$ & $\mathrm{P} 1$ ? \\
\hline 2001 May $7 \ldots \ldots$ & $13: 00$ & $12: 06$ & 1223 & 205 & $\mathrm{~N} 25, \mathrm{~W} 35$ & $\mathrm{nY}$ & $\mathrm{F} 2$ & 2001 Apr 2 & 12:00 & $11: 26$ & 992 & 80 & N17, W60 & yY & F1 \\
\hline 2001 Jun 15 . & $16: 00$ & $15: 56$ & 1701 & $\mathrm{H}$ & SW90(b) & yY & F1 & 2001 Apr 9 & 16:00 & $15: 54$ & 1192 & $\mathrm{H}$ & S21, W04 & yY & $\mathrm{N}$ \\
\hline 2001 Aug 9 & 19:00 & & & & S17, E19 & & & 2001 May 20. & 07:00 & $06: 26$ & 546 & 179 & S16, W90 & yY & $\mathrm{F} 2$ ? \\
\hline 2001 Aug $16 \ldots \ldots$ & 01:00 & $23: 54^{\mathrm{e}}$ & 1575 & $\mathrm{H}$ & Backside & $\mathrm{nY}$ & P1 & 2001 Jun 1 & 18:00 & $17: 30$ & 800 & 71 & W90(b) & $\mathrm{nN}$ & $\mathrm{F} 1$ \\
\hline 2001 Sep $15 \ldots \ldots$ & $12: 00$ & $11: 54$ & 478 & 130 & S21, W49 & yY & F1 & 2001 Jun 4 & 17:00 & $16: 30$ & 464 & 89 & N24, W59 & yN & $\mathrm{F} 1$ ? \\
\hline 2001 Sep $24 \ldots \ldots$ & $11: 00$ & $10: 30$ & 2402 & $\mathrm{H}$ & S16, E23 & nY & $\mathrm{F} 1$ & 2001 Oct 19 & 02:00 & 01:27 & 558 & 254 & N16, W18 & yY & $\mathrm{P} 1$ ? \\
\hline 2001 Oct 1 & $13: 00$ & $05: 30$ & 1405 & $\mathrm{H}$ & S20, W90 & $\mathrm{nY}$ & $\mathrm{F} 4$ & & & & & & & & \\
\hline 2001 Oct $19 \ldots \ldots$ & $17: 30$ & $16: 50$ & 901 & $\mathrm{H}$ & $\mathrm{N} 15, \mathrm{~W} 29$ & yY & P1 & & & & & & & & \\
\hline 2001 Oct 22 . & $17: 00$ & $15: 06$ & 1336 & $\mathrm{H}$ & S21, E18 & yY & F1 & & & & & & & & \\
\hline 2001 Nov $4 \ldots \ldots$ & $17: 00$ & $16: 35$ & 1810 & $\mathrm{H}$ & N06, W18 & yY & NS & & & & & & & & \\
\hline
\end{tabular}

${ }^{\text {a }}$ Speed in $\mathrm{km} \mathrm{s}^{-1}$

${ }^{\mathrm{b}}$ Width in degrees with $\mathrm{H}$ denoting full halos.

${ }^{\mathrm{c}}$ Type II radio burst.

${ }^{\mathrm{d}}$ Nature of interaction $(\mathrm{N}$, no interaction; NH, interaction with a preceding halo CME; NL, interaction with one of the legs of a preceding CME; NS, interaction with one or more western bright streamers).

${ }^{\mathrm{e}}$ Previous day.

${ }^{\mathrm{f}}$ Also had partial interaction.

few cases, there was no obvious interaction with a preceding CME, but there was clear interaction with one of the legs of a preceding CME (marked as NL) or with a bright western streamer (marked as NS). NH denotes interaction with a preceding halo. Events with no obvious interaction with any of these features are marked by $\mathrm{N}$ (including purely eastern interactions). Two examples of the height-time plots are shown in Figure 1: the 1997 November 6 event is preceded by an F1 interaction, while the 2001 October 1 event is preceded by an F4 interaction. We have used linear fits to the height-time plots in determining the height of intersection between the primary and preceding CMEs. If we use quadratic fits, we expect a slightly larger number of interactions. Columns (9)-(16) of
Table 1 have all the information for the minor SEP events. In the next section we describe the results of our analysis.

\section{RESULTS}

\subsection{Characteristics of the Primary CMEs}

The primary CMEs of the major SEPs were the fastest (average speed $\sim 1393 \mathrm{~km} \mathrm{~s}^{-1}$; see Fig. $2 a$ ), while those of the minor SEPs were slightly slower (average speed $\sim 927 \mathrm{~km} \mathrm{~s}^{-1}$; see Fig. 2b). All the primary CMEs had speeds exceeding the average speed $\left(\sim 450 \mathrm{~km} \mathrm{~s}^{-1}\right)$ of the general population of CMEs. The longitude distribution of the solar sources of the primary CMEs is similar for the major and minor SEPs (Figs. 

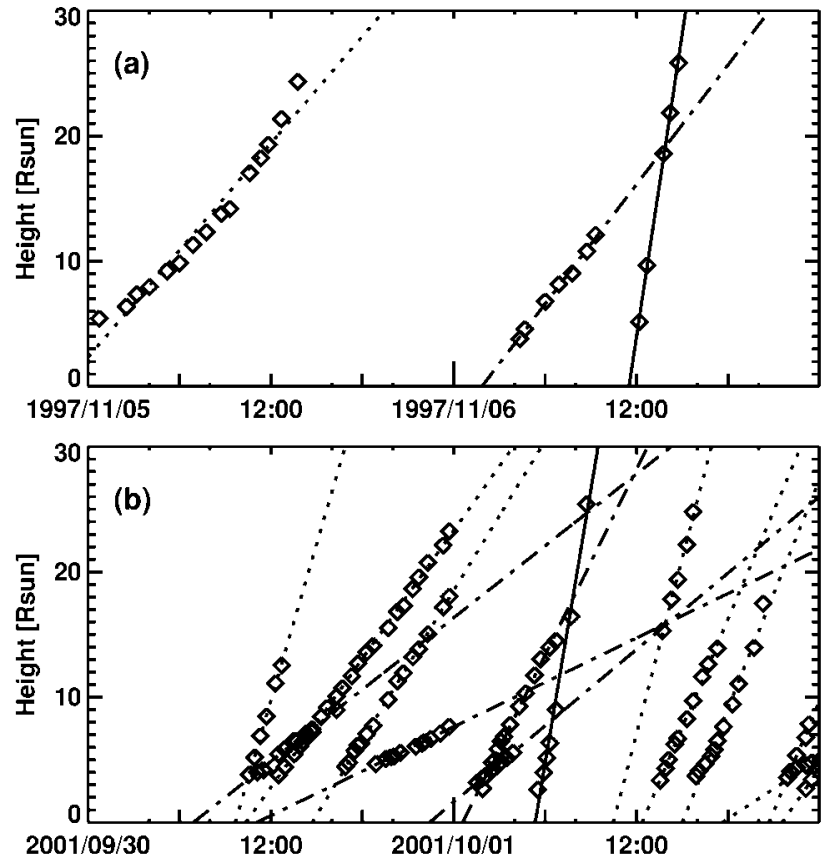

FIG. 1.-Height-time plots (diamonds: data points; lines: linear fits) of all the CMEs that preceded the primary CMEs (solid lines) within 2 days. CMEs interacting with the primary CMEs are shown by dot-dashed lines. Other CMEs occurring elsewhere and not interacting with the primary CMEs are shown by dotted lines. (a) The 1997 November 6 event with a single interaction (F1 in Table 1). (b) The 2001 October 1 event with multiple interactions (F4 in Table 1).

$2 c$ and $2 d$ ). All the solar sources were west of E46 ${ }^{\circ}$. The last bin $\left(90^{\circ}+\right)$ in Figures $2 c$ and $2 d$ containing the behind-thelimb events is the largest for both sets of SEPs. The longitude distribution is also somewhat different from the canonical distribution of gradual SEP events (Reames 1995), which extended all the way to the east limb. Table 1 (cols. [5] and [13]) shows that a major fraction of the primary CMEs were halos. In fact, the primary CMEs of $98 \%$ of the major SEPs and $87 \%$ of the minor SEPs had widths exceeding $100^{\circ}$ (see Table 2). Although
TABLE 2

SumMARY OF RESUlTS

\begin{tabular}{ccc}
\hline \hline Property & Major SEP & Minor SEP \\
\hline No. of events $\ldots \ldots \ldots \ldots \ldots \ldots \ldots \ldots \ldots$ & 43 & 39 \\
Average CME speed $\left(\mathrm{km} \mathrm{s}^{-1}\right) \ldots \ldots \ldots \ldots$ & 1393 & 927 \\
CME longitude $(\mathrm{deg}) \ldots \ldots \ldots \ldots \ldots \ldots \ldots$ & $>\mathrm{E} 32$ & $>\mathrm{E} 46$ \\
Width $>100^{\circ} \ldots \ldots \ldots \ldots \ldots \ldots \ldots \ldots \ldots$ & $41 / 42(98 \%)$ & $34 / 39(87 \%)$ \\
SEPs with CME interaction $\ldots \ldots \ldots \ldots$ & $35 / 42(83 \%)$ & $33 / 39(84 \%)$ \\
SEPs with all interactions $\ldots \ldots \ldots \ldots \ldots$ & $39 / 42(93 \%)$ & $37 / 39(95 \%)$ \\
Association with metric type II $\ldots \ldots \ldots$ & $30 / 42(71 \%)$ & $23 / 39(59 \%)$ \\
Association with DH type II $\ldots \ldots \ldots \ldots$ & $40 / 42(95 \%)$ & $22 / 39(56 \%)$ \\
Onset time difference $(\Delta t$ hr $) \ldots \ldots \ldots \ldots$ & 6.9 & 7.3 \\
Height of intersection $(R \odot) \ldots \ldots \ldots \ldots \ldots$ & 21 & 21 \\
P.A. overlap $(\Delta w$ deg $) \ldots \ldots \ldots \ldots \ldots \ldots$ & 50 & 53 \\
F/W CMEs with SEPs $\ldots \ldots \ldots \ldots \ldots \ldots$ & $26 / 52$ & $16 / 52$ \\
Average CME speed $\left(\mathrm{km} \mathrm{s}{ }^{-1}\right) \ldots \ldots \ldots$ & 1433 & 1178 \\
Width $>100^{\circ} \ldots \ldots \ldots \ldots \ldots \ldots \ldots \ldots \ldots$ & $25 / 26(96 \%)$ & $15 / 16(94 \%)$ \\
\hline
\end{tabular}

we cannot measure the actual widths of halo CMEs, we can say that the SEP accelerators expand rapidly to acquire a large angular size within the coronagraph field of view. Thus, the SEP-associated primary CMEs are very fast and very wide and occur west of $\mathrm{E} 45^{\circ}$.

\subsection{SEPs and CME Interaction}

Table 1 shows that a vast majority of the primary CMEs (35/42, or $83 \%$ for major SEPs, and $33 / 39$, or $84 \%$ for minor SEPs) interacted with one or more preceding CMEs. These percentages go up to $93 \%$ and $95 \%$ for major and minor events, respectively, when $\mathrm{NH}, \mathrm{NL}$, and NS interactions are included. Single interactions were most common, but there were also some multiple interactions. The preceding CMEs were mostly slow events (average speeds of 436 and $356 \mathrm{~km} \mathrm{~s}^{-1}$ for major and minor events, respectively; see Figs. $2 e$ and $2 f$ ). The P.A. overlap $(\Delta w)$ between the primary and the preceding CMEs is typically $\sim 50^{\circ}$ (Figs. $2 g$ and $2 h$ ). The preceding CMEs that interact with the primary CMEs depart typically a few hours earlier (average $\sim 7 \mathrm{hr}$; see Figs. $2 i$ and $2 j$ ). The heliocentric distance at which the leading edges of the primary and preceding CMEs intersect is $\sim 21 R_{\odot}$ (Figs. $2 k$ and $2 l$ ). Since CMEs have a finite thickness, the interaction must start much before

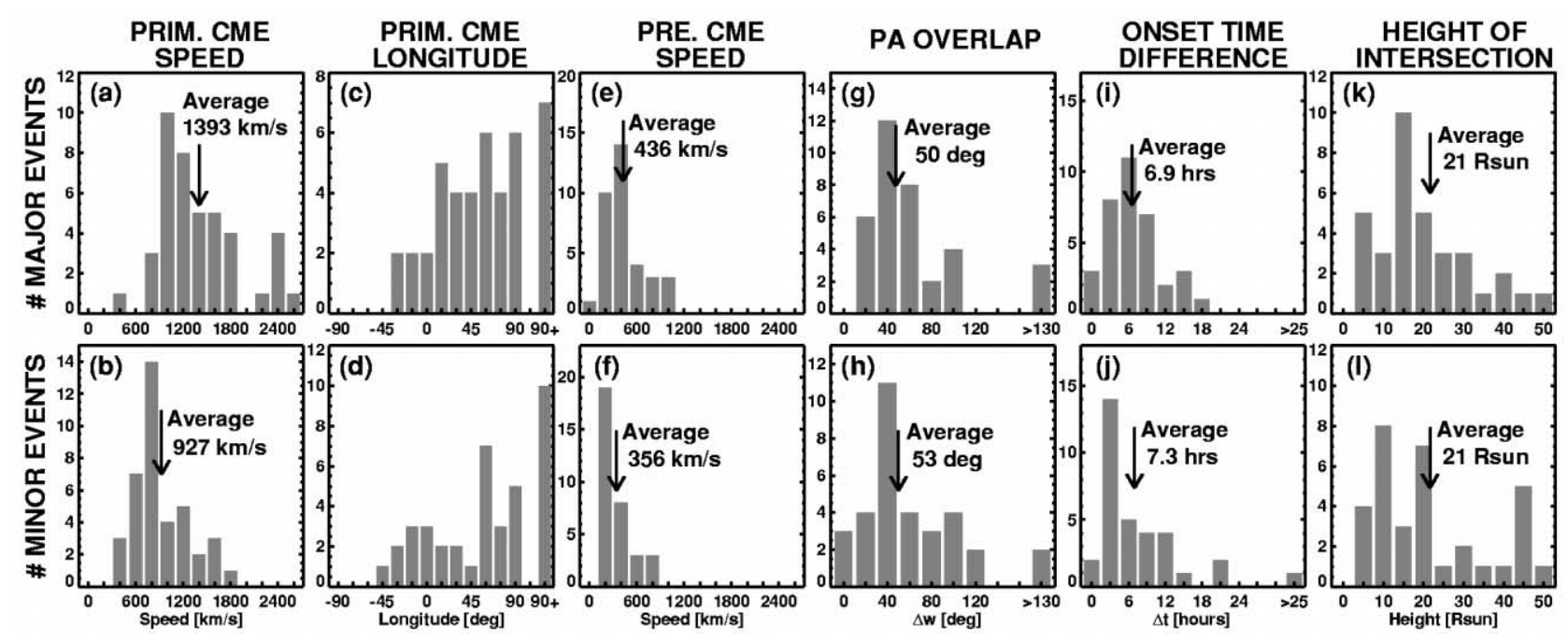

FIG. 2.-Properties of primary CMEs and interaction characteristics for major (top panel) and minor (bottom panel) SEP events. $(a, b)$ Speeds and $(c, d)$ source

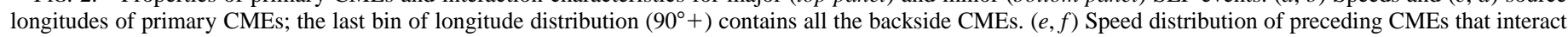

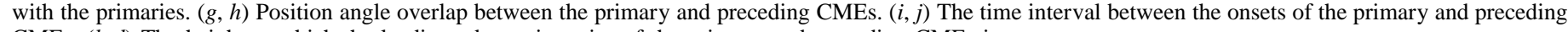
CMEs. $(k, l)$ The height at which the leading-edge trajectories of the primary and preceding CMEs intersect. 
the intersection of leading-edge trajectories. From the radio emission characteristics of interacting CMEs, Gopalswamy et al. (2001c) found that the interaction starts $\sim 1 \mathrm{hr}$ before the intersection of trajectories. Furthermore, when the height-time measurements are made at the P.A. of interaction rather than that of fastest motion in the CMEs (as was done here), the trajectories would intersect at lower heights.

\subsection{Inverse Study of Fast and Wide CMEs and SEPS}

One might wonder if the above result is simply due to the fact that CMEs are more frequent during solar maximum, so CME interaction must be commonplace. To show that this is not the case, we examined the CME interaction and SEP association for all the fast (speed greater than $900 \mathrm{~km} \mathrm{~s}^{-1}$ ) and wide (width greater than $60^{\circ}$ ) CMEs with a westward bias (halos and western hemisphere events) that occurred during the study period. We also required that the CME span must include P.A. $270^{\circ}$ to increase the likelihood of involving the Sun-Earth flux tube. There were 124 such CMEs, but many of them originated from behind the limb. The SEP association was not clear for some events owing to the enhanced SEP background from previous events, which we dropped. In order to develop a clean sample, we considered only the frontside, western hemisphere CMEs. This resulted in 52 fast and wide (F/W) CMEs, of which 42 were associated with SEPs (above the GOES threshold and unambiguously distinguishable as a separate event when the background is high); the remaining 10 (19\%) were not (see Table 2). Table 3 summarizes the SEP association and the extent of CME interaction for these CMEs. The numbers under the "Minor SEP" column include a few weaker events with $I_{p}<1$ pfu but above the GOES threshold; $I_{p} \geq 1$ pfu minor events are shown in parentheses. Interactions in the eastern hemisphere are unlikely to be relevant for SEP events, so we included four such events in the "No Interaction" category. We see that the number of SEPpoor CMEs with (6/10) and without (4/10) CME interaction is roughly the same. On the other hand, 35/42 (=83\%) of SEPassociated CMEs interacted with preceding CMEs, while only $7 / 42(=17 \%)$ did not. Considering just the full interactions, we see that only $2 / 10(=20 \%)$ of SEP-poor CMEs were preceded by CME interaction, compared to $33 / 42(=79 \%)$ for SEPassociated CMEs. We note that the SEP-poor CMEs had an average speed of $1220 \mathrm{~km} \mathrm{~s}^{-1}$, significantly higher than that of the minor-SEP CMEs and close to that of major-SEP CMEs in Table 1, with a similar comparison for SEP-associated F/W CMEs. Five of the SEP-poor CMEs were halos, and 8/10 ( $=80 \%$ ) had widths greater than $100^{\circ}$. Thus, the CME interaction seems to discriminate between the SEP-poor and SEP-associated ones since the speed and width of the two populations are comparable.

\subsection{Type II Radio Bursts and SEPs}

All but two $(40 / 42=95 \%)$ of the major SEP events were associated with DH type II bursts (see Table 1, cols. [7] and [15]). The DH type II bursts are known to be associated with faster and wider (hence more energetic) CMEs (Gopalswamy et al. 2001b). Shocks driven by these CMEs accelerate electrons, which in turn produce the radio bursts. The high degree of association between major SEP events and DH type II bursts suggests that the proton accelerators are also good electron accelerators. The association is somewhat poorer for the minor SEP events: only $22 / 39$, or $56 \%$, of the minor SEP events were associated with DH type II bursts. The metric type II burst association is poorer for both major (71\%) and minor (59\%)
TABLE 3

FAST AND Wide CMEs AND SEPs

\begin{tabular}{cccc}
\hline \hline Interaction & No SEP & Minor SEP & Major SEP \\
\hline No interaction .......... & 4 & $3(2)$ & 4 \\
Partial interaction $\ldots \ldots \ldots$ & 4 & $1(1)$ & 1 \\
Full interaction $\ldots . . . \ldots$ & 2 & $12(7)$ & 21 \\
\hline
\end{tabular}

Note. $-I_{p} \geq 1 \mathrm{pfu}$ minor events are shown in parentheses.

SEP events. The poor association between metric type II bursts and SEP events resembles a similar relationship between metric type II bursts and interplanetary shocks (Gopalswamy et al. 2001a). The type II radio burst association seems to be the only property that is significantly different for the major and minor SEP events. Interestingly, none of the SEP-poor F/W CMEs (see $\$ 3.3$ ) were associated with DH type II bursts.

\section{DISCUSSION AND CONCLUSIONS}

The primary result of this Letter is that a major fraction of the SEP events occur at times of CME interaction. Each SEP event is associated with a primary CME, which sweeps up one or more slower CMEs in the near-Sun interplanetary medium. The estimated height of intersection close to the Sun $\left(\sim 20 R_{\odot}\right)$ is significant because SEPs are thought to be released when the associated CME reaches a height of $\sim 5-15 R_{\odot}$ (Kahler 1994). The westward bias of the solar sources is consistent with the possibility that the Sun-Earth flux tube that carries SEPs is likely to be affected by the CME interaction. Furthermore, the huge angular widths of the SEP producers ensure that they intersect the ecliptic. Using a sample of fast and wide frontside CMEs, we were able to show that while the speeds, widths, and source locations of CMEs are important for predicting SEP events, the interaction with preceding CMEs proves to be another powerful discriminator.

The results presented in this Letter have important implications for theories of particle acceleration by shocks. The outermost structure of a fast CMEs is an MHD shock, which will first interact with the preceding CME. The shock has to pass through the inhomogeneous multithermal plasma (core, cavity, and frontal of the preceding CMEs). Thus, the shock has to accelerate the SEPs from the solar wind "contaminated" by the preceding CMEs, rather than from the quiet solar wind. On the basis of this, we suggest that the charge state composition of SEPs should rarely reflect the quiet solar wind conditions and that CME interaction may result in time-dependent, mixed impulsive-gradual signatures in SEP events. Mason et al. (1999) have attributed the mixed signatures to the "lingering flare superthermals" in the in-ecliptic interplanetary medium.

The interaction rather than the speed of the preceding CMEs seems to be important for the SEP production. This argues against the preacceleration of the seed particles except in a few cases where the preceding CME also drives a shock. The interaction seems to enhance the acceleration efficiency of the shock either because of the higher density material (of the preceding CMEs) injected into the shock or because of the trapping of particles in the closed loops of the preceding CMEs. Passage of shocks through extremely dense streamers located nearby may also have similar effects.

This research was supported by AFOSR and NASA's SR\&T, ISTP, and LWS programs. We thank the referee for many helpful comments. $S O H O$ is a project of international cooperation between ESA and NASA. 


\section{REFERENCES}

Bougeret, J.-L., et al. 1995, Space Sci. Rev., 71, 231

Brueckner, G. E., et al. 1995, Sol. Phys., 162, 357

Gopalswamy, N., Lara, A., Kaiser, M. L., \& Bougeret, J.-L. 2001a, J. Geophys. Res., 106, 25,261

Gopalswamy, N., Yashiro, S., Kaiser, M. L., Howard, R. A., \& Bougeret, J.-L. 2001b, J. Geophys. Res., 106, 29,219 . 2001c, ApJ, 548, L91
Gopalswamy, N., Yashiro, S., Kaiser, M. L., Howard, R. A., \& Bougeret, J.-L. 2002, Geophys. Res. Lett., 29(8), in press, 10.1029/2001GL013606 Kahler, S. W. 1994, ApJ, 428, 837 2001, J. Geophys. Res., 106, 20,947

Mason, G., et al. 1999, ApJ, 525, L133

Reames, D. V. 1995, Rev. Geophys. Suppl., 33, 585

—. 1999, Space Sci. Rev., 90, 413 